\title{
Forelimb Bone Morphology and its Association with Foraging Ecology in Four Families of Neotropical Bats
}

\author{
Mariano S. Sánchez ${ }^{1}$ (D) Luz V. Carrizo $^{1}$ (D) \\ Accepted: 14 October 2020 \\ (C) Springer Science+Business Media, LLC, part of Springer Nature 2020
}

\begin{abstract}
The form-function paradigm postulates the existence of a correlation between form and function; a remarkable example of this is the transformation of the forelimb of bats into wings. The wings of bats are complex structures made of membranes, muscles, tendons, cartilages, bones, and joints. Forelimb bones are critical elements of wings because they form an essential part of the alar mass, serve to support soft tissue, and resist deformations. Only a few studies have attempted to detect relationships between morphology and ecology of the forelimb bones in bats in an assemblage context. Here, we explored ecomorphological patterns of 180 specimens belonging to 22 genera and 37 species of four families, occurring in northern Argentina. We quantitatively characterized bones and analyzed their relation with wing shape, as described by aerodynamic parameters, and ecological habits in a phylogenetic context. For the analyses, we used eight ecological categories, five wing variables that included three aerodynamic indexes, 29 linear measurements of the bones (humerus, radius, and scapula), and lengths of digits III and V. As expected, specimens distribution in morphospace showed that species tend to be segregated on the basis of size and morphology. Interestingly, Molossidae and Lasiurini occupy nearby areas in the morphospace, which can reveal patterns of functional convergences between them. A significant correlation between wing shape and morphology of forelimb bones was detected, showing a link between the latter and aerodynamic variables. We observed a remarkable morphological variation in the structures of the proximal and distal ends of the humerus, the proximal end of the radius, and in the acromion of the scapula. Our results highlight that these variations reflect the degrees of specialization in different modes of flight and ecological habits. We also observed that phylogeny explained in part the morphological patterns and shows a strong covariation with ecological habits, finding different phylogenetic patterns among the main lineages (Molossidae, Vespertilionidae, and Phyllostomidae).
\end{abstract}

Keywords Aerodynamic parameters $\cdot$ Ecomorphology $\cdot$ Morphofunctional patterns $\cdot$ Phylogeny $\cdot$ Wing shape

\section{Introduction}

Birds and bats are the only extant vertebrates that have evolved powered flight (Norberg 1990, 1994). Flying locomotion permits migration by the travel of long distances, foraging in inaccessible places and over large areas, and feeding by the capture of flying insects (Norberg 1994).

Supplementary Information The online version of this article (https:// doi.org/10.1007/s10914-020-09526-5) contains supplementary material, which is available to authorized users.

Mariano S. Sánchez

marianoseb.sanchez@gmail.com

1 Instituto de Biología Subtropical (IBS), Consejo Nacional de Investigaciones Científicas y Técnicas (CONICET) and Laboratorio de Genética Evolutiva, Universidad Nacional de Misiones (UNaM), Félix de Azara 1552, N3300LQF Posadas, Misiones, Argentina
However, flying locomotion is energetically very expensive and, thus, may represent an evolutionary constraint to several morphological, physiological, and ecological features (Norberg and Rayner 1987; Voigt and Holderied 2012).

The form-function paradigm postulates the existence of a correlation between form and function (Radinsky 1987); a remarkable example of this is the transformation of the forelimb of bats into wings (Radinsky 1987). From an aerodynamic perspective, variations in the shape and size of wings can be related to different types of flight (Rayner 1986; Norberg 1990, 1994). Features such as surface-to-body size ratio, wingtip shape, wingspan, and their relationship to wing width are commonly used to describe the shape of the wing and different aspects of flight performance such as agility, speed, maneuverability, and energetic costs (Norberg 1990, 1994; Voigt and Holderied 2012; Marinello and Bernard 2014). These characters have been associated with the foraging 
Table 1 Examined species in this study and examples of wing shape illustrating qualitative differences among species belonging to different ecological categories. The abbreviations for each species are indicated in brackets and bold

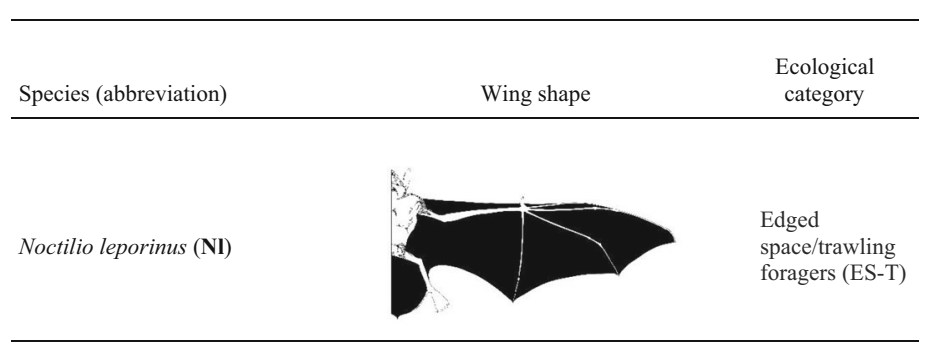

Diaemus youngii" (Dy)

Desmodus rotundus" (Dr)

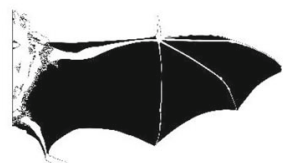

Narrow space/ ground foragers (NS-G)

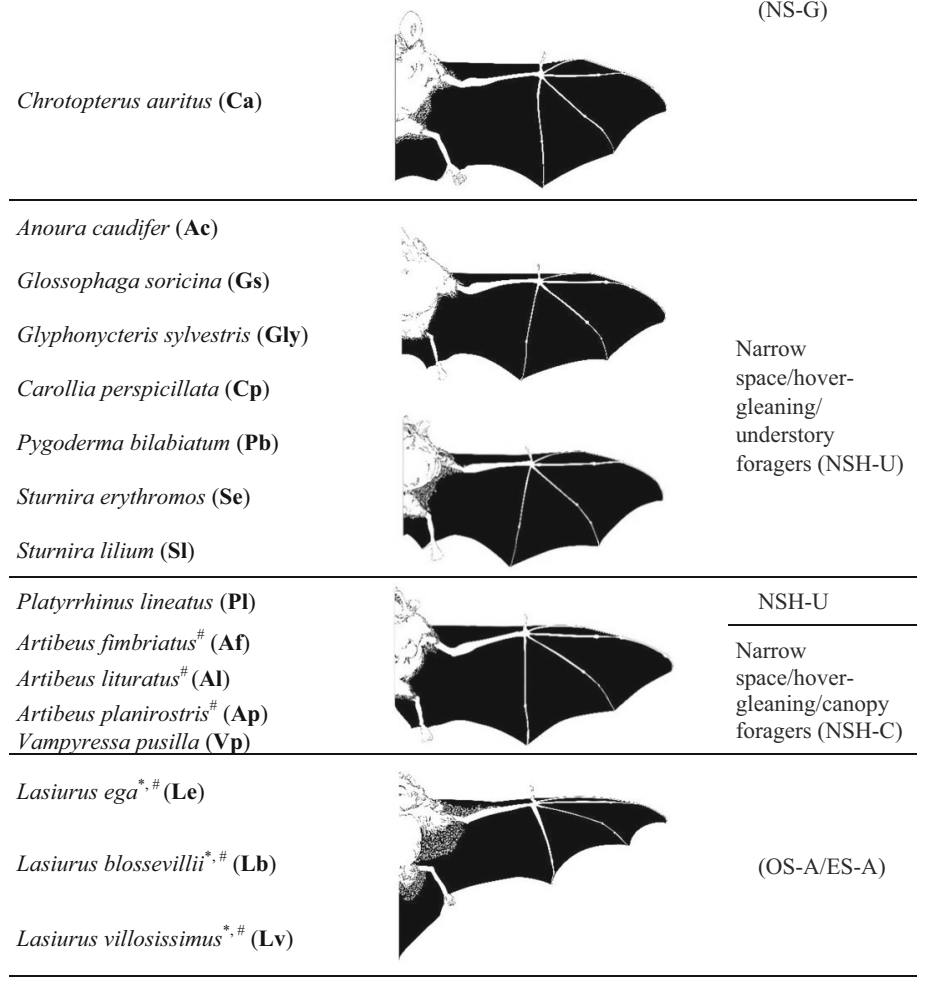

Eptesicus furinalis $(\mathbf{E f})$

Myotis albescens

Myotis nigricans

Myotis dinellii (My spp.)

Myotis ruber

Myotis keaysi

Myotis riparius

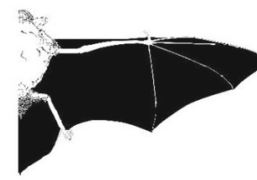

Edged

space/aerial

foragers (ES-A)

Molossops temminckii (Mte)

ES-A

Cynomops planirostris ${ }^{\#}$ (Cyp)

Cynomops abrasus ${ }^{\#}$ (Cya)

Eumops glaucinus ${ }^{*}, \#$ (Eug)

Eumops perotis", \# (Eup)

Eumops bonariensis ${ }^{\#}$ (Eub)

Eumops patagonicus ${ }^{\#}$ (Epa)

Molossus molossus ${ }^{\#}$ (Mom)

Molossus rufus" (Mor)

Nyctinomops macrotis ${ }^{*}, \#$ (Nym)

Tadarida brasiliensis ${ }^{*}, \#$ (Tb)

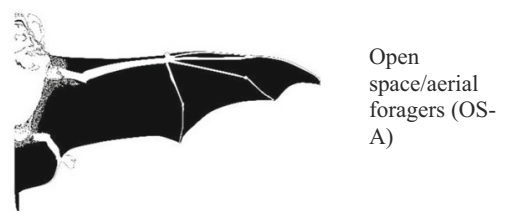

Migratory species (*) (MIG); Commuting species (\#) (COM); all Myotis species were grouped on "My spp." abbreviation 
strategy of bats from an ecomorphological perspective (e.g., Norberg and Rayner 1987; Marinello and Bernard 2014).

Several studies confirmed that the flight of bats is very complex, as a consequence of changes in wing shape during flapping flight (Riskin et al. 2008; Swartz et al. 2012; Bahlman et al. 2016). The wings of bats are made of membranes, muscles, tendons, cartilages, bones, and joints, with a tridimensional configuration that changes through the wingbeat cycle (Swartz et al. 2007, 2012; Riskin et al. 2008). Forelimb bones are critical elements of a wing that form an essential part of the alar mass, serve to support soft tissue, and resist deformations (Swartz et al. 2007, 2012; Panyutina et al. 2015). These elements have been analyzed only for some species, based on comparative descriptions (Vaughan 1966; Vaughan and Bateman 1970; Walton and Walton 1970; Altenbach 1979; Hermanson and Altenbach 1985; Panyutina et al. 2015) or by biomechanical, experimental, and evolutionary approaches (e.g., Swartz et al. 1992; Riskin et al. 2008; Swartz and Middleton 2008; Bahlman et al. 2016). Only a few authors have attempted to detect some relationship between forelimb bone morphology and ecology in bats in an assemblage context (e.g., López-Aguirre et al. 2019). This lack of study is particularly evident for Neotropical regions like northern Argentina, where a rich bat fauna occurs and assemblages are characterized by species with a wide variety of foraging and feeding habits (see Sandoval et al. 2010; Sánchez 2016).
In this study we explored, under an ecomorphological approach (Wainwright 1991), the morphological pattern of the forelimb bones of 37 species of bats of four families, occurring in northern Argentina. We quantitatively characterized bones and analyzed their relation with wing shape, as described by aerodynamic parameters, and ecological habits in a phylogenetic context. We expected that the morphological diversity of the bones of the forelimb are strongly associated with ecological habits due to different mechanical demands imposed by different flight and foraging behaviors. As many aerodynamic parameters of the wing and ecological habits apparently emerged early in the evolutionary history of bat lineages (e.g., Sánchez and Giannini 2018; Amador et al. 2020), we also expected that phylogeny has an important effect in morphological diversity and in structuring the functional morphospace.

\section{Materials and Methods}

Sample We analyzed bones from the forelimb and shoulder girdle from 180 specimens belonging to 22 genera and 37 species of four families of Neotropical bats (Noctilionidae, Phyllostomidae, Vespertilionidae, and Molossidae) that span a wide range of foraging behavior and flying types (Table 1). The taxonomic sample was restricted to those voucher specimens that were collected through successive field research

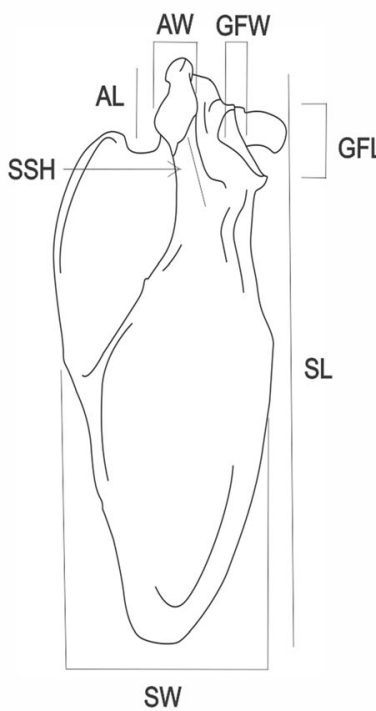

SW

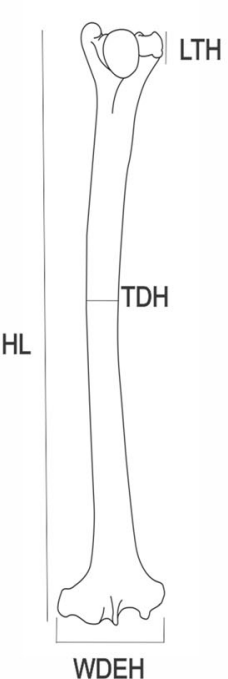

WDEH

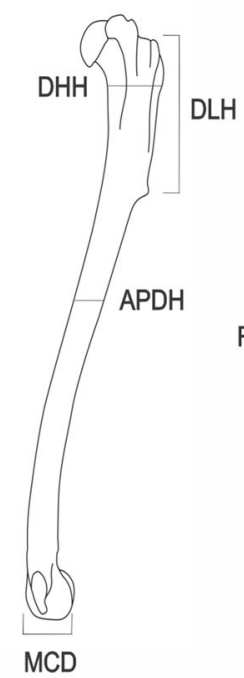

condyle (capitulum) width; LCL, lateral condyle (capitulum) length; MCD, medial condyle (trochlea) deep; WDASH, width of the distal articular surface of the humerus; WDEH, width of the distal end of the humerus; SPL, styloid process length (spine entepicondyle); GTH, greater tubercle height; LTH, lesser tubercle height. Radius: RHW, radius head width; RHL, radius head length; RL, radius length; TDR, transverse diameter radius; APDR, anteroposterior diameter radius. Descriptions of the measures in Online Resource 3 
projects (e.g., Sandoval et al. 2010; Sánchez 2011, 2016; Sánchez et al. 2019). Voucher specimens were all adults of both sexes preserved as skin, skull, and postcranial skeleton, and are deposited in the systematic collections listed in Online Resource 1. Collecting of the specimens was made following the ethical guidelines approved by the American Society of Mammalogists (see Sikes et al. 2016) and the recommendations of the Administración de Parque Nacionales (APN), Ministerio de Ecología y RNR de Misiones, and Dirección de Recursos Naturales de la Provincia de Corrientes (Permits $\mathrm{N}^{\circ}:$ 32-07, 074, 028, 102, 001, 001-19, $002,845)$. For the analyses, we preferably included specimens in which both wing and osteological variables were measured, although in some cases, only osteological data were available. The latter drawback was solved by estimating aerodynamic parameters (hereafter wing variables) in individuals of the same species and sex with a similar mass and forearm size. In our matrices, this procedure was applied only in 14 individuals of nine species (highlighted as * in Online Resource 2).

Morphological Data We photographed the ventral view of the wing from freshly euthanized animals using a NIKON D3200 digital camera to describe the wing outline (hereafter wing shape). To photograph specimens, we followed the standard methodologies used in Camargo and de Oliveira (2012). We recorded six measurements of the wing that were used to estimated: aspect ratio (AR), wing loading (WL), tip shape index $\left(I_{\text {tip }}\right)$. These latter, plus wingspan $(B)$, and wing area (S) were used as descriptors of the wing shape. A detailed description of these measures and the aerodynamic parameters is provided in Online Resource 3. Measurements of the wings were taken from the digital images using ImageJ 1.50.e software (Rasband 2018). Body mass was recorded using a spring scale Pesola TM $(100 \pm 0.5 \mathrm{~g})$.

The osteological dataset included 29 linear measurements of bones (humerus, radius, and scapula), corresponding to lengths and midshaft diameters of the bones, as well as lengths of various functionally important muscular insertions sites (Fig. 1, Online Resource 3). Additionally, measurements of the hand were made (length of the digits III and V). For the terminology of bones and processes, we followed the descriptions of Vaughan and Bateman (1970) and Panyutina et al. (2015). The measurements were performed under a binocular stereoscopic microscope using a digital caliper DIGIMESS $®( \pm 0.01 \mathrm{~mm})$. Average and standard deviation of each variable by species are provided in Online Resource 2.

Ecological Categories Bats were grouped into eight ecological categories (functional groups) according to habitat use, foraging behavior, or mobility capacity (see Denzinger et al. 2018 and references therein, Table 1, and Online Resource 3). The diet preference (e.g., fruits, nectar, or arthropods) does not act as a good predictor of foraging behavior and was not considered, as it can generate redundancy and background noise in the classification (Denzinger et al. 2018). Bat species were assigned into each category based on the available literature and field observations (see Online Resource 3).

Statistical Analyses First, we performed a size-correction of all morphological variables using geometric mean transformations (the ratio between individual's values and the geometric mean of the corresponding variables; e.g., Giménez and Giannini 2016). These values were used in subsequent analyses as size-corrected morphological variables. Values belonging to each ecological category were transformed into the arcsine of the square root after running analyses.

A Principal Components Analysis (PCA) was performed upon a correlation matrix of morphometric data (36 wing and osteology variables), in order to determine the patterns of morphofunctional variation among the 37 bat species from four families. On the PCA ordination diagram, we traced minimum polygons joining specimens from predominant ecological habits or family, and marked each species. Besides, two PCA were run to summarize the morphometric variation of each dataset separately (Online Resource 4). We performed a Partial Least Squares Analyses (PLS) for evaluating the morphological integrations between wing shape and skeletal variables (see Rohlf and Corti 2000). For the PLS analyses, Block 1 was represented by seven wing variables and Block 2 by 29 linear bone measurements.

A Redundancy Analyses (RDA, ter Braak and Šmilauer 2002) was run to estimate the correlation between morphological variables and ecological categories. In our case, RDA used morphological data as the main matrix and eight ecological categories as explanatory variables. Significant ecological categories $(\alpha<0.01)$ were assessed individually using 9999 unrestricted Monte Carlo permutations. A forward stepwise selection from the ecological categories matrix was then preformatted (see ter Braak and Šmilauer 2002).

We estimated phylogenetic effects on the morphological variability as described above in the PCA, using Canonical Phylogenetic Ordination (CPO), a comparative phylogenetic method derived from linear regression models (Giannini 2003). This analysis was done because bat species may have variable degrees of statistical dependence due to common ancestry (Giannini 2003). The main matrices were the same as in PCA or RDA, whereas the explanatory variables consisted of a set of binary variables coding clade membership for each bat species. We used the phylogenetic trees from Medina et al. (2014) and Amador et al. (2018) as a reference to construct our tree matrix, which were pruned to consider only the species of bats included in our study (see Fig. 5). Clades' significance was individually assessed using 9999 unrestricted 

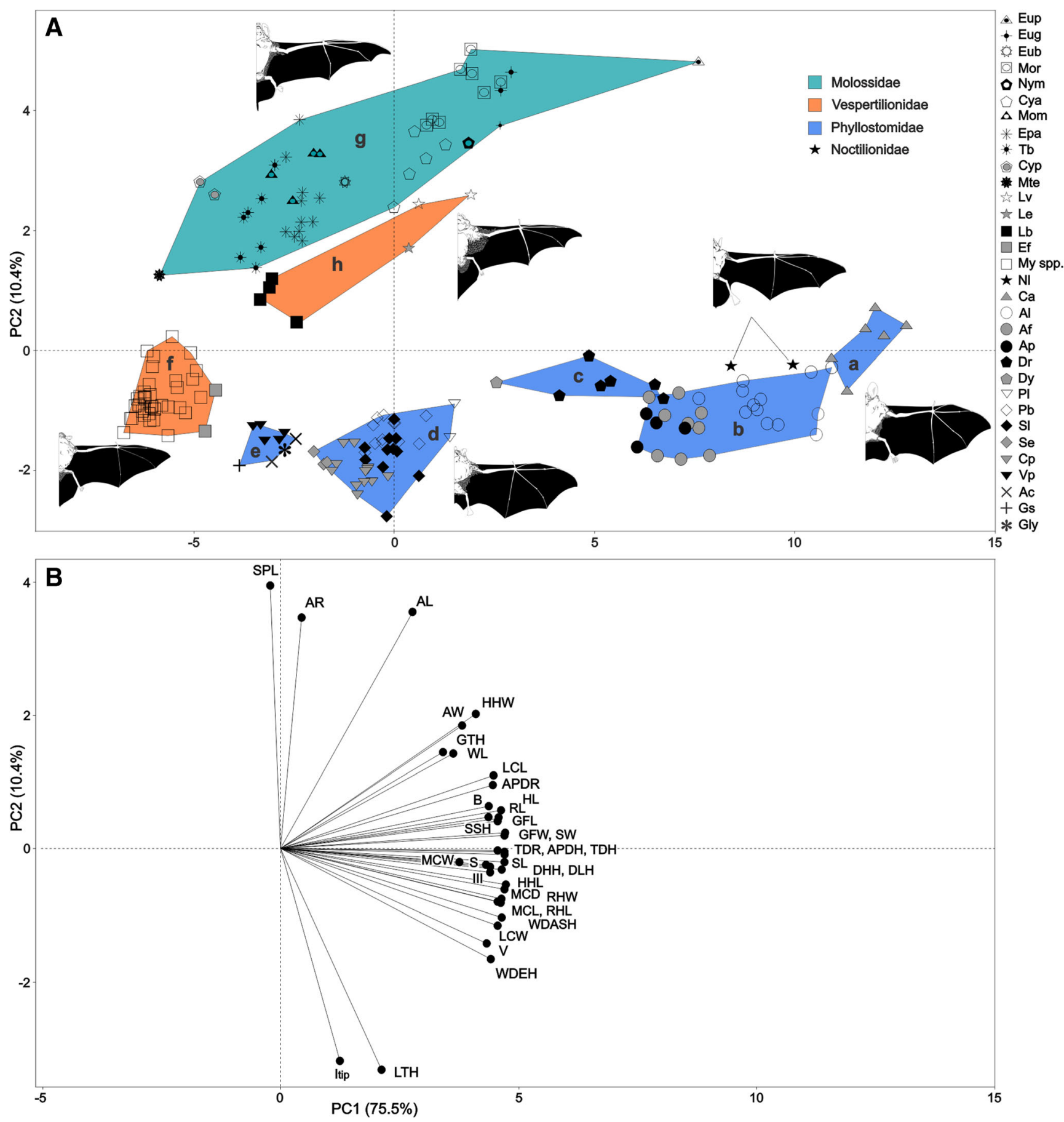

Fig. 2 Ordination diagram of the Principal Components Analysis (PCA). A Segregation of the specimens using all dataset (wing and forelimb bone variables). Polygons include specimens from ecological categories: (a) and (c), narrow space/ground foragers; (b), narrow space/hover-gleaning/canopy foragers; (d) and (e), narrow space/hover-gleaning/

Monte Carlo permutations testing at $\alpha<0.01$. A further selection of clades from the tree matrix was performed to determine clades that best explain total variation attributable to phylogenetic structure, i.e., those clades that best explained the variation between species by common ancestry excluding redundancy (Giannini 2003). understory foragers; (f), edged space/aerial foragers; (g) and (h), Open space/aerial foragers and migratory. B Vectors shown the strength of correlation of each variable to the plane of PC1 and PC2. See Fig. 1 for abbreviations

A partial Canonical Phylogenetic Ordination (pCPO, Giannini 2003) was used to test covariation between ecological categories and phylogeny, and separate the variation into three components: percentages corresponding to ecological categories alone, clades alone, and their covariations. RDA, CPO, and pCPO analyses were carried out using CANOCO 4.5 (ter 


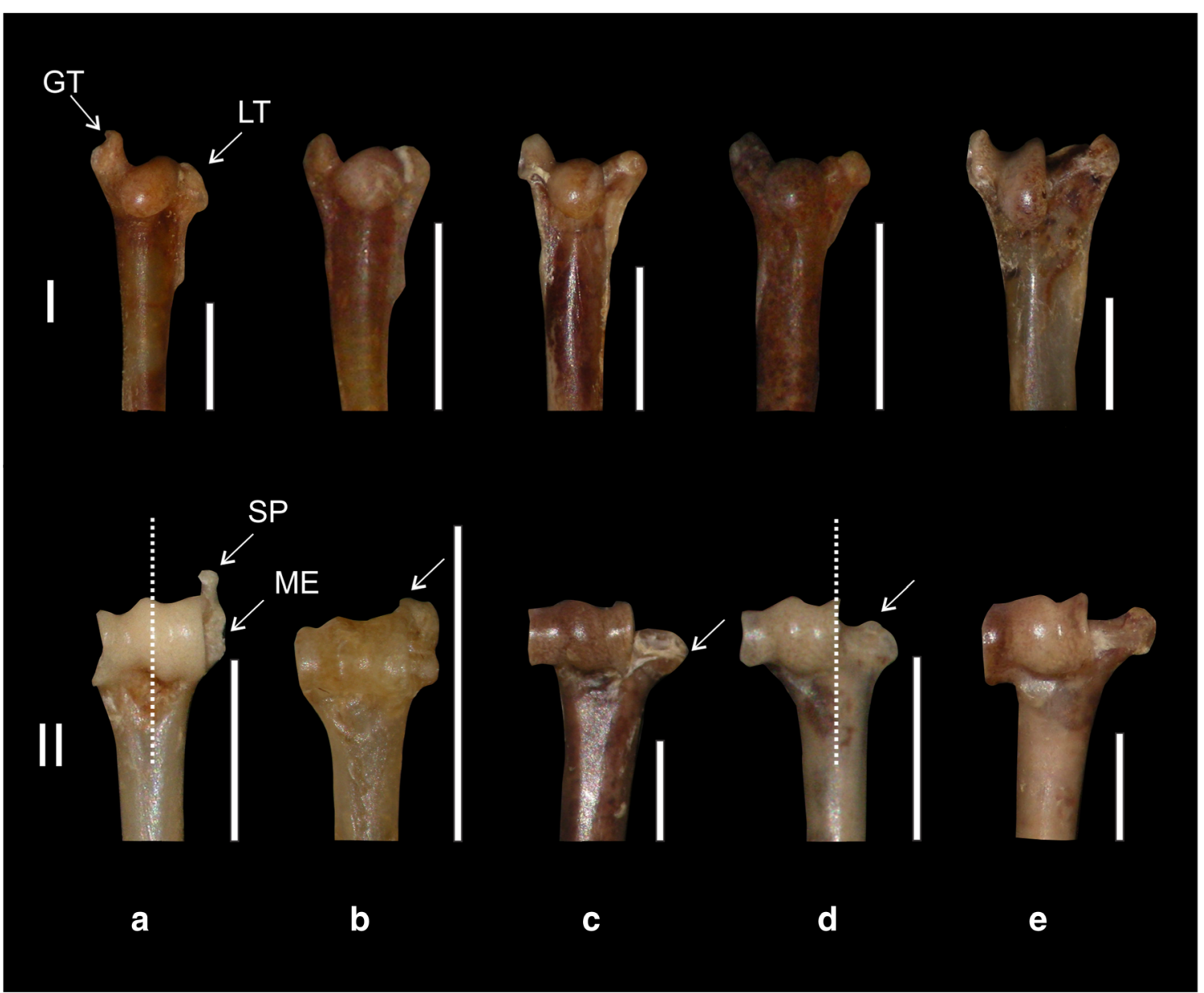

Fig. 3 Humerus in several bats. I, posterior view of the proximal part; II, anterior view of the distal part. a Eumops glaucinus; $\mathbf{b}$ Myotis nigricans; $\mathbf{c}$ Artibeus lituratus; $\mathbf{d}$ Carollia perspicillata; and e Noctilio leporinus. The greater tubercle (GT) is high and well developed in Eumops, while in the remaining species it is similar in size to the lesser tubercle (LT). The

Braak and Šmilauer 2002). PCA was performed using FactoMineR and factoextra R packages (see Kassambara 2017), PLS was performed using 'pls2B' function in the Morpho R package 2.6 (Schlager 2017), and significance was assessed using permutations test (10,000 round). These analyses were run in R version 3.6.0 (R Development Core Team 2019).

The datasets generated and/or analyzed during the current study available from the corresponding author on reasonable request.

\section{Results}

\section{Morphological Patterns}

The PCA using the dataset with all morphometric variables showed that the first two principal components explained $85.9 \%$ of the total variation in the morphological space (PC1 $75.5 \%$ and PC2 10.4\%, respectively; Online Resource 4). spinous process (SP) presents different morphologies, being long and well developed in Eumops, poorly developed in Myotis and Carollia, and absent in Artibeus. Noctilionoidea species (c, d and e) have good development of the medial epicondyle (ME) and the articular surface is laterally displaced from the humeral shaft (dash line)

Most variables were positively and highly correlated with PC1, whereas only some of them were positively or negatively correlated with PC2 (AR, SPL, AL, Itip, and LTH; Fig. 2B). The species dispersion on $\mathrm{PC} 1$ showed a continuous decreasing gradient from the positive to the negative end of the axis, from larger (e.g., Chrotopterus and Noctilio) to small-sized species (e.g., Myotis spp., Molossops, and Vampyressa). Species segregation on PC2 showed a morphological gradient from bats with narrowest wings and pointy wingtips at the end of the positive axis (Molossidae + Lasiurus spp.) to bats with broad wings and triangle to rounded wingtips at the end of the negative axis (Phyllostomidae, Noctilio, Eptesicus, and Myotis spp.; Fig. 2A).

Complete segregation emerged from the joint analyses of PC1 and PC2 (Fig. 2). Molossidae and Lasiurus spp. dominate the positive extreme of PC2 (polygon (g) and (h); Fig. $2 \mathrm{~A})$. These groups are characterized by relatively narrow and pointed wings (AR higher and lesser $\mathrm{I}_{\text {tip }}$ ), relatively short fifth finger, a long acromion (AL), the humeral head is elongated 
Table 2 Results of Redundancy Analyses for four families of bats and eight ecological categories

\begin{tabular}{lllll}
\hline Test & Ecology features & $F$ & $p$ & V\% \\
\hline Individuals & ES-A & 83.39 & $\mathbf{0 . 0 0 0 1}$ & 43.1 \\
& NSH-C & 42.64 & $\mathbf{0 . 0 0 0 1}$ & 26.1 \\
& NS-G & 40.13 & $\mathbf{0 . 0 0 0 1}$ & 24.8 \\
& COM & 26.70 & $\mathbf{0 . 0 0 0 1}$ & 17.6 \\
& OS-A & 19.67 & $\mathbf{0 . 0 0 0 1}$ & 13.4 \\
& ES-T & 5.83 & 0.0093 & 4.3 \\
& NSH-U & 5.63 & 0.0095 & 4.1 \\
& MIG & 5.25 & 0.0118 & 3.9 \\
& ES-A, OS-A & 61.49 & $\mathbf{0 . 0 0 0 1}$ & 66.2 \\
& NSH-U & 102.17 & $\mathbf{0 . 0 0 0 1}$ & 91.6 \\
& NSH-C & 18.55 & $\mathbf{0 . 0 0 0 1}$ & 96.2 \\
& MIG & 10.15 & $\mathbf{0 . 0 0 3 1}$ & 97.6 \\
\hline
\end{tabular}

Significant $p$-values at $\alpha<0.01$ are indicated in bold; $F$, statistic value of permutations test; V\%, percentage variance explained. See Table 1 for abbreviations

anteroposteriorly (HHW; Fig. 3), the lesser tubercle is low with respect to the humeral head (LTH; Fig. 2B, 3), the distal end of the humerus is narrow (WDEH, WDASH, and LCW), and SPL is well developed.

Positive and negative ends of PC1 and PC2 are defined by several groups characterized mainly by rounded wings (high $\mathrm{I}_{\text {tip }}$ ) and the lesser tubercle well developed (LTH; Figs. 2, 3). The groups that composed the three first polygons, (a) (Chrotopterus), (b) (Artibeus), and (c) (Desmodus and Diaemus), and $N$. leporinus present broad wings with a long fifth finger, with a wider distal end and distal articular surface of the humerus (WDEH, WDASH, LCW, MCL, MCD, and MCW), and a well-developed radial head (RHL and RHW). A fourth group, the polygon (d) (Pygoderma, Sturnira, Platyrrhinus, and Carollia; Fig. 2A) presents the most rounded wingtips ( $\mathrm{I}_{\text {tip }}>>1$; see Fig. $2 \mathrm{~B}$ ), a short acromion $(\mathrm{AL})$, a lesser tubercle that is subequal to greater tubercle (LTH; Figs. 2B, 3), and an underdeveloped or absent spinous process (SPL). These traits are shared with small phyllostomids from polygon (e) (Vampyressa, Glossophaga, Anoura, and Glyphonycteris). Finally, polygon (f) (Myotis spp. and $E$. furinalis) has triangular wingtips $\left(\mathrm{I}_{\text {tip }} \approx 1\right)$, SPL is always present but small, and LTH little developed (Figs. 2B, 3).

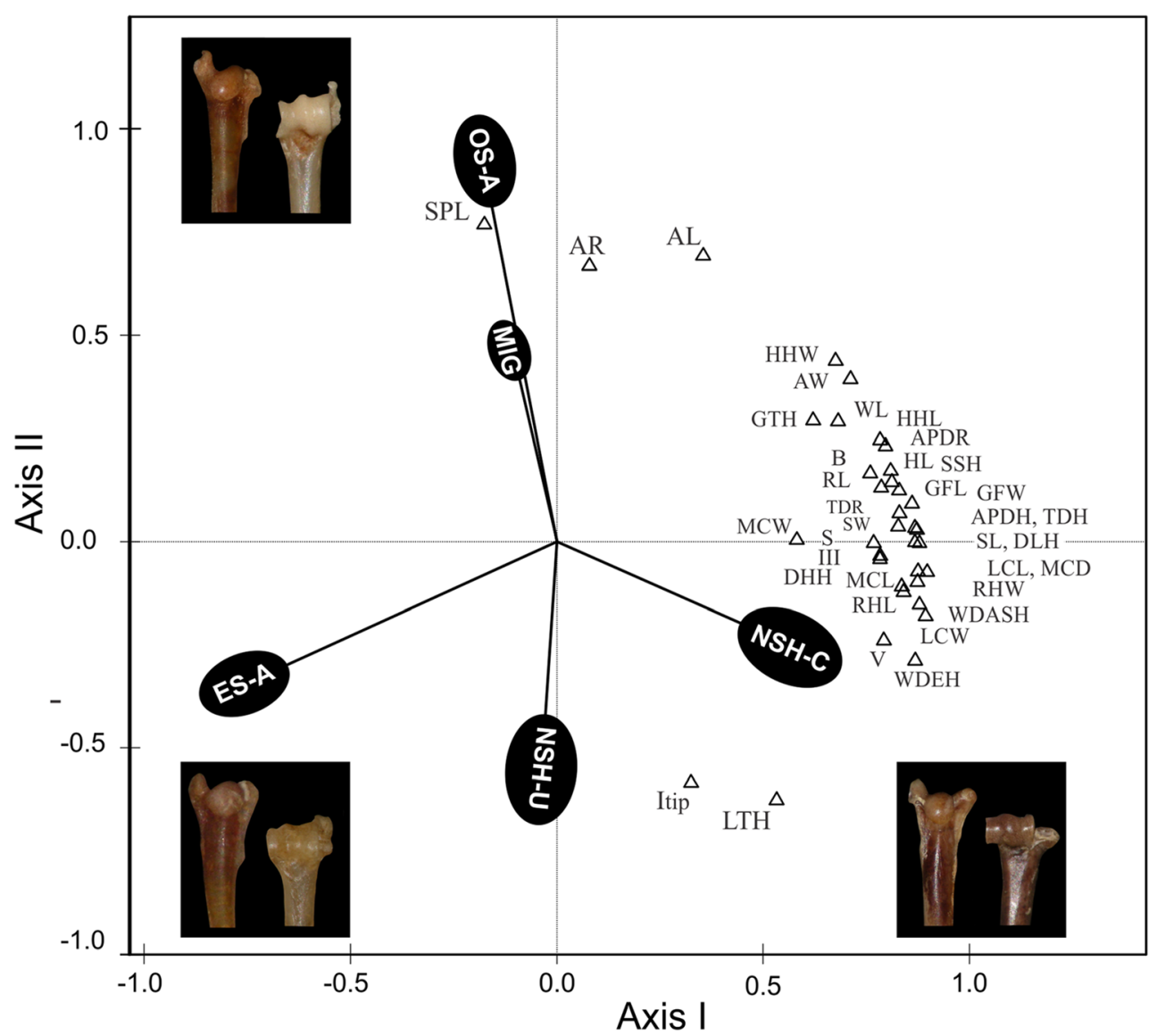

Fig. 4 Correlation among morphological traits and ecological categories as shown by RDA. First two axes explain $97.6 \%$ of the total morphological variation associated with ecological variables and only five groups were recovered. See abbreviations in Table 1. Note the difference in the morphology of the proximal and distal end of the humerus in the different ecological groups 
Table 3 Results of Canonical Phylogenetic Ordination for morphometric datasets

\begin{tabular}{|c|c|c|c|c|}
\hline Test & Clade & $F$ & $p$ & $\mathrm{~V} \%$ \\
\hline \multirow[t]{20}{*}{ Individuals } & 40 & 89.15 & 0.0001 & 36.9 \\
\hline & 60 & 89.15 & 0.0001 & 36.9 \\
\hline & 73 & 83.77 & 0.0001 & 35.5 \\
\hline & 61 & 79.04 & 0.0001 & 33.1 \\
\hline & 41 & 71.14 & 0.0001 & 31.6 \\
\hline & 42 & 68.52 & 0.0001 & 30.8 \\
\hline & 44 & 63.40 & 0.0001 & 29.1 \\
\hline & 45 & 59.918 & 0.0001 & 27.9 \\
\hline & 65 & 59.75 & 0.0001 & 27.8 \\
\hline & 72 & 57.95 & 0.0001 & 27.3 \\
\hline & 63 & 55.56 & 0.0001 & 26.4 \\
\hline & 74 & 51.61 & 0.0001 & 24.9 \\
\hline & 70 & 44.27 & 0.0001 & 22.0 \\
\hline & 68 & 37.19 & 0.0001 & 19.1 \\
\hline & 66 & 32.77 & 0.0001 & 17.2 \\
\hline & 50 & 18.77 & 0.0001 & 10.6 \\
\hline & 51 & 15.33 & 0.0002 & 8.8 \\
\hline & 46 & 11.16 & 0.0002 & 6.6 \\
\hline & 53 & 8.11 & 0.0025 & 4.8 \\
\hline & 62 & 6.34 & 0.0047 & 3.8 \\
\hline \multirow[t]{12}{*}{ Forward stepwise } & $40^{*}, 73^{*}$ & 48.28 & 0.0001 & 52.8 \\
\hline & $42 *$ & 54.55 & 0.0001 & 66.6 \\
\hline & $66^{*}$ & 67.00 & 0.0001 & 78.9 \\
\hline & $64 *$ & 40.16 & 0.0001 & 85.0 \\
\hline & $62 *$ & 27.62 & 0.0001 & 88.5 \\
\hline & $55^{*}$ & 19.35 & 0.0001 & 90.8 \\
\hline & $41^{*}$ & 17.54 & 0.0001 & 92.7 \\
\hline & $52^{*}$ & 15.47 & 0.0001 & 94.2 \\
\hline & $59^{*}$ & 10.34 & 0.0001 & 95.2 \\
\hline & $67^{*}$ & 8.91 & 0.0001 & 96.0 \\
\hline & $48^{*}$ & 7.05 & 0.0006 & 96.7 \\
\hline & $61^{*}$ & 8.30 & 0.0004 & 97.3 \\
\hline
\end{tabular}

Significant $p$-values for each clade at $\alpha<0.01$ are indicated in bold; $F$, statistic value of permutations test; V\%, percentage variance. Numbers with asterisks represent groups that are integrated in the optimal model (after forward stepwise selection of variables). Clades are numbered as in Fig. 5. A table with the results for all clades is presented in Online Resource 6

Partial least-squares analyses (PLS) of the wing shape and bone morphology show a high correlation between blocks $(r=0.916, p=0.0001$ after 10,000 rounds of permutation tests; Online Resource 4) that indicates a high association between wing shape and the different variables of the bones of forelimbs. The PLS analyses show that the first two vectors explain $99 \%$ of the covariation between both datasets (Online Resource 4 and 5).

\section{Ecomorphological Relationships}

After Monte Carlo permutations test $(\alpha<0.01)$, the RDA model retains five ecological categories that jointly explained $97.6 \%$ of the morphological variation $(F=10.15, p=0.003$; Table 2; Fig. 4). On the positive side of the first axis, narrow space canopy foragers (NSH-C; $F=18.55, p=0.0001$ ) were defined by V, WDEH, LCW, WDASH, RHW, RHL, and MCL (Fig. 4). On the positive side of the second axis, migrators (MIG; $F=10.15$, $p=0.003$ ) and open space/aerial foragers (OS-A; $F=61.49, p=$ 0.0001 ) were defined by SPL, AR, AL, and low values of $\mathrm{I}_{\text {tip }}$ and LTH. On the negative side of the second axis, narrow space understory foragers (NSH-U; $F=102.17, p=0.0001$ ) were defined by $\mathrm{I}_{\text {tip }}$ and LTH. Lastly, edged space/aerial foragers (ES-A; $F=83.39, p=0.0001$ ) were not defined by any particular variable (Fig. 4).

\section{Phylogenetic Patterns}

The $\mathrm{CPO}$ results indicated that most tree partitions were individually significant at $\alpha<0.01$ (Table 3, Online Resource 6), all of which were important to explain morphological variation on the wing shape and forelimb bones. After the selection of clade, 13 partitions were included in the reduced tree matrix that together explained, without redundancy, $97.3 \%$ of the morphological variation $(F=8.3, p=0.0004$; Table 3 ). The models successively incorporated the larger lineage of the insectivorous bats (Vespertilionidae + Molossidae; clade 40 in Fig. 5), followed by clades 73 (Artibeus spp.), 42 (Myotis spp.), 66 separating the basal unranked clade Nullicauda from the other phyllostomid species, 41 (Vespertilionidae), 48 (Lasiurus spp.), and 61 (separating Phyllostomidae from Noctilionidae lineage). Also, some recent clades were recovered (clades 64, 62, 55, 52, 59, and 67; see Fig. 5 and Table 3). In the partial CPO, we detected a small fraction of the morphological variance of the main matrix that was associated exclusively with ecological variables $(0.3 \%$, Online Resource 6).

\section{Discussion}

Our results confirm an important relationship between forelimb bone morphology, wing shape, and ecological habits as proposed in previous studies (e.g., Vaughan 1959, 1970a, b; Altenbach 1979; Norberg 1990, 1994; López-Aguirre et al. 2019). Aerodynamic variables were also associated with forage habits as predicted in previous works (see a detailed treatment in Norberg 1990, 1994; Marinello and Bernard 2014). Besides, our analyses show that phylogeny explained in part the morphological patterns and show a strong covariation with ecological habits. In this section, we will discuss mainly the most important morphological patterns of the forelimb bones and their functional implications in the different ecological groups. 
Fig. 5 Cladogram of four Neotropical families of bats, based on Medina et al. (2014) and Amador et al. (2018). Thick lines and arrows indicate significant clades individually and after stepwise method, respectively, in $\mathrm{CPO}$. The colors correspond to each family of bats. Note that Vespertilionidae + Molossidae (clade 40) and Noctilionidae (star) were also significant and are indicate with thick black lines

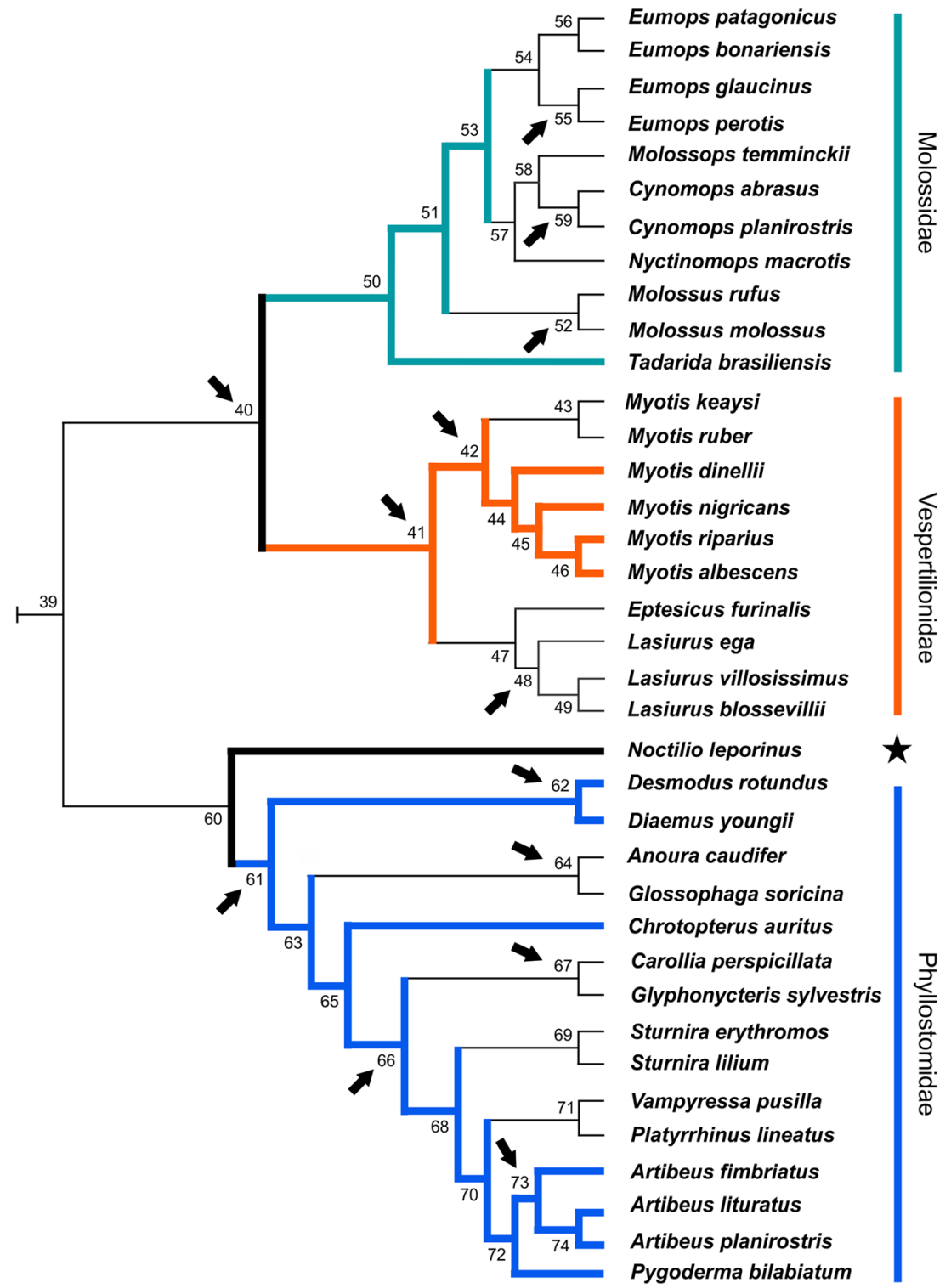

\section{Morphological Patterns and Ecological Habits}

The morphology of the forelimb bones varies considerably between the species of our taxonomic sample. Notably, we observed a remarkable morphological variation in the structure of the proximal and distal ends of the humerus, the proximal end of the radius, and in the scapular acromion. Our results are consistent with previous studies that stress that these variations reflect, in part, degrees of specialization in different modes of flight (e.g., Vaughan 1966; Vaughan and Bateman 1970; Schliemann and Schlosser-Sturm 1999; Simmons and Geisler 1998).

Species that forage in open space (Molossidae and Lasiurini) tend to have an elongated humerus head and a lesser tubercle poorly developed with respect to the greater tubercle. These structures interact with the scapula forming a specialized shoulder joint with secondary articular surfaces, as described by
Schliemann and Schlosser-Sturm (1999). During flight, wing bones are under different pressures that are transmitted by the membrane and muscles, pronating the wing in the shoulder joint at the downstroke (Swartz et al. 1992). This type of joint limits the movements of humeral rotation, restricting its free movement (Schliemann and Schlosser-Sturm 1999; Panyutina et al. 2015). Interestingly, molossids occupy an extreme morphospace among bats, including its shoulder joint (Schliemann and Schlosser-Sturm 1999). Thus, a high specialization of these structures could be related to an increased stabilization, preventing the dislocation of the gleno-humeral joint and limit pronatory movement of the humerus during fast and enduring flight.

Other relevant information obtained from our analyses, which define molossids and vespertilionids, are the narrow distal end of the humerus, reduced medial epicondyle, articular 
surface (medial and lateral condyles) limited by well-marked ridges and grooves (non-spherical) and aligned with the humeral shaft (Fig. 3). Hand et al. (2009) described this type of configuration in mystacinids and proposed that this morphology leads to rigid articulation between the humerus and radius, which was associated with fast and enduring flight species such as Eumops and Molossus (Vaughan 1959, 1970b; Hand et al. 2009). The humerus of the open space foragers is also characterized by a well-developed spinous process, especially in molossids (Fig. 3). Originating on the latter structure is the $\mathrm{m}$. flexor carpi ulnaris whose action depends on the position of the radius and elbow; so, when the elbow flexes, the spinous process forms a wide angle with the radius increasing the distance between this process and the distal wing (see Vaughan and Bateman 1970). Vaughan (1970a) suggested that this arrangement helps govern the tautness of the plagiopatagium and the extension of the chiropatagium during flight, while Hand et al. (2009) interpreted this arrangement as a mechanical advantage to flex the hand during terrestrial locomotion in Mystacina. Finally, a large acromion with an evident lateral projection is a condition observed in many molossids (see Vaughan 1959; Walton and Walton 1970; Simmons and Geisler 1998). This morphology indicates the development of the muscles that elevate and rotate the humerus (e.g., m. acromiodeltoideus; Vaughan 1959; Panyutina et al. 2015).

In species that foraging in narrow spaces (Phyllostomidae), the distal end of the humerus is wide and has an articular surface laterally displaced from the humeral shaft, the spinous process is small or absent, and the medial epicondyle is well developed (Fig. 3). According to Vaughan (1959), this morphological pattern is related to a rearrangement of the forearm musculature ( $\mathrm{m}$. flexor carpi ulnaris and $\mathrm{m}$. flexor digitorum profundus), which migrates from the spinous process to the base and medial surface of the ulna or the medial epicondyle (as in Macrotus, Hipposideros, and Pteropus; see Vaughan 1959; Panyutina et al. 2015). In this configuration, the origin of muscles is near to the center of rotation of the elbow joint, and it could imply a loss of the mechanical advantage that was proposed by other authors (Vaughan 1959; Vaughan and Bateman 1970; Hand et al. 2009).

Understory foragers (Fig. 4) are mainly defined by having rounded humeral head, and the lesser tubercle well-developed, being equal to the greater tubercle in some species (e.g., C. perspicillata, P. bilabiatum, G. sylvestris, and G. soricina). This morphology is associated with a higher capacity of movement of the shoulder joint and a greater rotational stability of the humerus during downstroke by actions of the $\mathrm{m}$. subscapularis that inserts in the lesser tubercles (Vaughan 1959; Hermanson and Altenbach 1983, 1985; Panyutina et al. 2015). Electromyography activity of the $\mathrm{m}$. subscapularis in Antrozous and Artibeus showed its importance in the stabilization of the gleno-humeral joint and the fine control of the rotation of the humerus (Hermanson and Altenbach 1983, 1985).
This characteristic may be especially important in species that practice slow and hover-gleaning flights such as Carollia and Glossophaga.

In the canopy forager, the fifth finger is longer with respect to the other ecological categories and the distal end of the humerus is especially wide, linked primarily to an increase in the width of the lateral condyle. Broad wings are determined by the length of the fifth finger, which increases the wing area. These conditions are of great importance for large species like Artibeus or Chrotopterus, which can fly carrying heavy weights in their mouths, such as large fruits or small vertebrates (see Norberg 1994). Other features that define this group are the significant development of the distal articular surface of the humerus and the radial head, characters shared with sangivorous and carnivorous bats (D. rotundus, D. youngii, and C. auritus). Moreover, the distal articular surface of the humerus is characterized by a central spherical capitulum (rounded; Fig. 3). This morphology contrasts with the rigid articulation of molossids and vespertilionids, and could be associated with increased pronation and supination (see Hand et al. 2009). This could be important in species that practice true walking gait such as vampire bats, or for species that use wings during fruit handling, suspensive and roosting behavior (Altenbach 1979; Vandoros and Dumont 2004; Hand et al. 2009).

In species foraging in edged space (Myotis and Eptesicus), the humeral head is rounded, lesser and greater tubercles present similar developments with respect to the head of the humerus. As a general Bauplan in mammals, a rounded humeral head allows better rotational movements at the gleno-humeral joint (see Flores and Díaz 2009, and references therein). Bats with this condition tend to have a humerus with a greater range of movement (Vaughan 1959; Panyutina et al. 2015). Besides, the muscles inserted in the tuberosity present facultative activity depending upon the flight maneuver being performed (Hermanson and Altenbach 1983, 1985). Altogether, it could indicate that this morphology is associated with an increase in maneuverability required to forage near to vegetation. The spinous process is present but poorly developed, indicating that $\mathrm{m}$. flexor carpi ulnaris is inserted near the center of rotation of the elbow joints, lowering the lever arm system as described by Vaughan (1959).

\section{Historical Processes}

Phylogenetic comparative analyses suggest that the segregations in the morphospace among ecological groups of bats are associated with historical processes. The first significant group recovered in the final model separates the Vespertilionidae and Molossidae from the other lineages. We also observed a large dichotomy in Vespertilionidae between Myotis and Lasiurini, which showed contrasting morphotypes and aerodynamic parameters of wings (Fig. 2). 
On the one hand, Myotis has aerodynamic parameters that allow them to perform slow and maneuverable flights. This feature originated in extant bats (Palaeochiropteryx) and remained stable in Chiroptera phylogeny until the ancestor of Vespertilionidae ca. 30 mya (Amador et al. 2020), suggesting an ancestral morphotype of wings in Myotis. On the other hand, we observed that Lasiurini shows aerodynamic parameters and forelimb bone morphology related to Molossidae, as shown by the species segregation in morphospace. This wing shape is associated with fast and economic flight, typical of migratory species or open space foragers (Norberg 1994). The cladogenetic event that originated Lasiurini ca. 19.8 mya (Amador et al. 2018) could be interpreted as the emergence of a new morphotype that represents an invasion of new adaptive zones on the morphospace between Vespertilionidae and can evidence patterns of functional convergences with molossids.

In the Molossidae, some recent tree partitions were important to explaining the morphofunctional space. We firstly recovered a clade that separates large from small species of Eumops, followed by Molossus and Cynomops lineages. Larger species of Eumops diverged from the main tree partitions about ca. 9.3 mya, whereas Molossus and Cynomops clades diverged ca. 4.0 and 1.0 mya, respectively (Amador et al. 2018). Giménez and Giannini (2016) also found a hard split in the morphospace defined by the skull between larger Eumops and the remaining molossids. In our results, Eumops perotis is the largest species and represents a new size of wings, reflected in the significant increase of the wingspan, humerus and radius lengths, elongated humerus head, and a very long and wide acromion. These features were associated with long and sustained forager flight (Vaughan 1959; Norberg 1994) and could indicate a niche partitions with respect to medium-size molossids. Interestingly, E. perotis can forage for $6-8 \mathrm{~h}$ continuously and travel a relatively long distance from roost to feeding areas (58 km round trip; Best et al. 1996; Siders et al. 1999). In contrast, some medium-size species with short wings, such as Cynomops abrasus, leave colonies a little before sunset to feed, returning to the nightroosts in approximately $30 \mathrm{~min}$ (Sánchez pers. obs.).

The morphofunctional structure of the Phyllostomidae was statistically associated with major clades of the tree. Our phylogenetic effect analyses firstly recovered the tree partition that contains all the predominantly frugivores (Nullicauda), followed by the tree partitions where phyllostomids diverged from Noctilionoidea. Besides, recent clades were recovered, such as Artibeus, Desmodontinae, Glossophaginae, and Glyphonycteris + Carollia. These groups, particularly Nullicauda, experienced a pronounced shift in the diversification rate (Rojas et al. 2016), which was associated with diet specializations (Sánchez and Giannini 2018) and skull evolution (Rossoni et al. 2019). Our results indicate that wing shape and forelimb bone morphology in phyllostomids probably remained stable in the main lineages of the tree by ca. 11.2 mya (estimated from Amador et al. 2018), and these showed some changes in specific lineages. Rossoni et al. (2019) demonstrated that the cranial covariance structure and morphological differentiation in phyllostomids remained conserved on a broader phylogenetic scale, with multiple peaks adaptive on specific clades, and that the invasion of new adaptive zones drove these patterns. Our results suggest that wing morphology could have followed a similar evolutionary pattern but in a marginal way due to redundancies on the mechanical requirements of flight among different trophic groups.

Acknowledgments We especially thank to Guillermo Cassini, Néstor Toledo, and Sergio Vizcaíno for inviting us to participate in this tribute to L.B. Radinsky. We thank the curator of the Colección Mamíferos Lillo (CML) RM Barquez. Thank also to Juan M. Ferro and Alberto Taffarel for helping with the first version of the manuscript and Román Sánchez for helping with figures edition and for the drawings of the bones and bat wings. Two anonymous reviewers significantly improved the scope and quality of the manuscript.

Funding MSS was funded by PICT2013-2759 and PICT2016-0608 and LVC by PICT2015-2864.

\section{Compliance with Ethical Standards}

Conflict of Interest The authors declare that they have no conflict of interest.

\section{References}

Altenbach JS (1979) Locomotor morphology of the vampire bat, Desmodus rotundus. Am Soc Mammal Spec Publ 6:1-137

Amador LI, Almeida FC, Giannini NP (2020) Evolution of traditional aerodynamic variables in bats (Mammalia: Chiroptera) within a comprehensive phylogenetic framework. J Mammal Evol 27:549561.

Amador LI, Moyers Arévalo L, Almeida CF, Catalano SA, Giannini PG (2018) Bat systematics in the light of unconstrained analyses of a comprehensive molecular supermatrix. J Mammal Evol 25:37-70

Bahlman JW, Price-Waldman RM, Lippe HW, Breuer KS, Swartz SM (2016) Simplifying a wing: diversity and functional consequences of digital joint reduction in bat wings. J Anat 229:114-127

Best TL, Mark Kiser W, Freeman PW (1996) Eumops perotis. Mammal Species 534:1-8

Camargo NF, de Oliveira HFM (2012) Sexual dimorphism in Sturnira lilium (Chiroptera, Phyllostomidae): can pregnancy and pup carrying be responsible for differences in wing shape? PLoS One 7: e49734

Denzinger A, Tschapka M, Schnitzler H-U (2018) The role of echolocation strategies for niche differentiation in bats. Can J Zool 96:171181

Flores DA, Díaz MM (2009) Postcranial skeleton of Glironia venusta (Didelphimorphia, Didelphidae, Caluromyinae): description and functional morphology. Zoosyst Evol 85:311-339

Giannini NP (2003) Canonical phylogenetic ordination. Syst Biol 52: 684-695

Giménez AL, Giannini NP (2016) Morphofunctional segregation in molossid bat species (Chiroptera: Molossidae) from the South American Southern Cone. Hystrix Ital J Mammal 27:1-11 
Hand SJ, Weisbecker V, Beck RMD, Archer M, Godthelp H, Tennyson AJD, Worthy TH (2009) Bats that walk: a new evolutionary hypothesis for the terrestrial behaviour of New Zealand's endemic mystacinids. BMC Evol Biol 9:169

Hermanson JW, Altenbach JS (1983) The functional anatomy of the shoulder of the pallid bat, Antrozous pallidus. J Mammal 64:62-75

Hermanson JW, Altenbach JS (1985) Functional anatomy of the shoulder and arm of the fruit-eating bat Artibeus jamaicensis. J Zool 205: $157-177$

Kassambara A (2017) Practical guide to principal component methods in R: PCA, M (CA), FAMD, MFA, HCPC, factoextra. STHDA (http:// www.sthda.com). Accessed 22 September 2020

López-Aguirre C, Wilson LAB, Koyabu D, Tan Tu V, Hand SJ (2019) Decoupled morphological and biomechanical evolution and diversification of the wing in bats. EcoEvoRxiv. https://doi.org/10. 32942/osf.io/k3y5f

Marinello MM, Bernard E (2014) Wing morphology of Neotropical bats: a quantitative and qualitative analysis with implications for habitat use. Can J Zool 92:141-147

Medina CE, Gregorin R, Zeballos H, Zamora HT, Moras LM (2014) A new species of Eumops (Chiroptera: Molossidae) from southwestern Peru. Zootaxa 3878:19-36

Norberg UM (1990) Vertebrate Flight: Mechanics, Physiology, Morphology, Ecology and Evolution. Springer-Verlag, Berlin, $291 \mathrm{pp}$

Norberg UM (1994) Wing design, flight performance, and habitat use in bats. In: Wainwright PC, Reilly SM (eds) Ecological Morphology: Integrative Organismal Biology. University of Chicago Press, Chicago, pp 205-239

Norberg UM, Rayner JMV (1987) Ecological morphology and flight in bats (Mammalia: Chiroptera): wing adaptations, flight performance, foraging strategy and echolocation. Philos Trans R Soc Lond B 316 (1179):335-427

Panyutina AA, Korzun LP, Kuznetsov AN (2015) Flight of Mammals: From Terrestrial Limbs to Wings. Springer, London, 296 pp

R Development Core Team (2019) R: a language and environment for statistical computing. Foundation for Statistical Computing, Vienna. Available at: http://www.R-project.org

Radinsky LB (1987) The Evolution of Vertebrate Design. University of Chicago Press, Chicago, 197 pp

Rasband WS (2018) ImageJ. U. S. National Institutes of Health, Bethesda, https://imagej.nih.gov/ij/

Rayner JMV (1986) Vertebrate flapping flight mechamisms and aerodynamics, and the evolution of flight in bats. In: Natctigall W(ed) Bioma Report no.5: Bat flight. Gustav Fisher, Stuttgart, pp 27-74

Riskin DK, Willis DJ, Iriarte-Díaz J, Hedrick TL, Kostandov M, Chen J, Laidlawd DH, Breuer KS, Swartz SM (2008) Quantifying the complexity of bat wing kinematics. J Theor Biol 254:604-615

Rohlf FJ, Corti M (2000) Use of two-block partial least squares to study covariation in shape. Syst Biol 49:740-753

Rojas D, Warsi OM, Dávalos LM (2016) Bats (Chiroptera: Noctilionoidea) challenge a recent origin of extant Neotropical diversity. Syst Biol 65:432-448

Rossoni DM, Costa BMA, Giannini NP, Marroig G (2019) A multiple peak adaptive landscape based on feeding strategies and roosting ecology shaped the evolution of cranial covariance structure and morphological differentiation in phyllostomid bats. Evolution 73-5:961-981

Sánchez MS (2011) Interacción entre murciélagos frugívoros y plantas en las selvas subtropicales de Argentina. PhD Disertation, Universidad Nacional de Tucumán, Tucumán

Sánchez MS (2016) Structure of three subtropical bat assemblages (Chiroptera) in the Andean rainforests of Argentina. Mammalia 80:11-19

Sánchez MS, Giannini NP (2018) Tropic structure of frugivorous bats in the Neotropics: emergent patterns in evolutionary history. Mammal Rev 48:90-107
Sánchez MS, Labaroni CA, Castellanos Insuasti FX, Baldo D (2019) First record of Glyphonycteris sylvestris Thomas, 1896 (Chiroptera: Phyllostomidae: Glyphonycterinae) for Argentina, with comments on its karyotype. Mastozool Neotrop 26:420-429

Sandoval ML, Sánchez MS, Barquez RM (2010) Mammalia, Chiroptera Blumenbach, 1779: new locality records, filling gaps, and geographic distribution maps from northern Argentina. Check List 6:64-70

Schlager S (2017) Morpho and rvcg-shape analysis in R. In: Zheng G, Li S, Szekely G (eds) Statistical Shape and Deformation Analysis. Academic Press, London, pp 217-256

Schliemann H, Schlosser-Sturm E (1999) The shoulder joint of the Chiroptera - morphological features and functional significance. Zool Anz 238:75-86

Siders MS, Rabe MJ, Snow TK, Yasuda K (1999) Long foraging distances of two uncommon bat species (Euderma maculatum and Eumops perotis) in northern Arizona. In: van Riper C III, Stuart MA (eds) Proceedings of the Fourth Biennial Conference of Research on the Colorado Plateau, Flagstaff, pp 113-122

Sikes RS, and the Animal Care and Use Committee (2016) 2016 guidelines of the American Society of Mammalogists for the use of wild mammals in research and education. J Mammal 97:663-688

Simmons NB, Geisler JH (1998) Phylogenetic relationships of Icaronycteris, Archaeonycteris, Hassianycteris, and Palaeochiropteryx to extant bat lineages, with comments on the evolution of echolocation and foraging strategies in Microchiroptera. Bull Am Mus Nat Hist 253:4-180

Swartz SM, Bennett MB, Carrier DR (1992) Wing bone stresses in free flying bats and the evolution of skeletal design for flight. Nature 359:726-729

Swartz SM, Iriarte-Diaz J, Riskin DK, Breuer KS (2012) A bird? A plane? No it's a bat: an introduction to the biomechanics of bat flight. In: Gunnell GF, Simmons NA (eds) Evolutionary History of Bats: Fossils, Molecules, and Morphology. Cambridge University Press, Cambridge, pp 317-352

Swartz SM, Iriarte-Diaz J, Riskin DK, Song A, Tian X, Willis DJ, Breuer KS (2007) Wing structure and the aerodynamic basis of flight in bats. Proc. of the 45th AIAA Aerospace Science Meeting, Reno

Swartz SM, Middleton KM (2008) Biomechanics of the bat limb skeleton: scaling, material properties and mechanics. Cells Tissues Organs 187:59-84

ter Braak CJF, Šmilauer P (2002) CANOCO, Reference Manual and CanoDraw for Windows User's Guide: Software for Canonical Community Ordination (Version 4.5). Microcomputer Power, Ithaca

Vandoros JD, Dumont ER (2004) Use of the wings in manipulative and suspensory behaviors during feeding by frugivorous bats. J Exp Zool A 301A:361-366

Vaughan TA (1959) Functional morphology of three bats: Eumops, Myotis, Macrotus. Univ Kansas Publ Mus Nat Hist 12:1-153

Vaughan TA (1966) Morphology and flight characteristics of molossid bats. J Mammal 47:249-260

Vaughan TA (1970a) The muscular system. In: Wimsatt WA (ed) Biology of Bats. Academic Press, New York, pp 140-193

Vaughan TA (1970b) The skeletal system. In: Wimsatt WA (ed) Biology of Bats. Academic Press, New York, pp 97-138

Vaughan TA, Bateman GC (1970) Morphology of the forelimb of mormoopid bats. J Mammal 51:217-235

Voigt CC, Holderied MW (2012) High manoeuvring costs force narrowwinged molossid bats to forage in open space. J Comp Physiol B 182:415-424

Wainwright PC (1991) Ecomorphology: experimental functional anatomy for ecological problems. Am Zool 31:167-194

Walton DW, Walton GM (1970) Post-cranial osteology of bats. In: Slaughter BH, Walton DW (eds) About Bats: A Chiropteran Biology Symposium. Southern Methodist University Press, Dallas, pp 93-126 